Relations industrielles

Industrial Relations

\title{
Le secret en matière d'inventions, André Bertin, Éditions du Tambourinaire, Entreprise Moderne d'Édition, Paris, 1965. 157 pages.
}

\section{Fernand Blais}

\section{Volume 21, numéro 2, 1966}

URI : https://id.erudit.org/iderudit/027684ar

DOI : https://doi.org/10.7202/027684ar

Aller au sommaire du numéro

Éditeur(s)

Département des relations industrielles de l'Université Laval

ISSN

0034-379X (imprimé)

1703-8138 (numérique)

Découvrir la revue

Citer ce compte rendu

Blais, F. (1966). Compte rendu de [Le secret en matière d'inventions, André Bertin, Éditions du Tambourinaire, Entreprise Moderne d’Édition, Paris, 1965. 157 pages.] Relations industrielles / Industrial Relations, 21(2), 301-302. https://doi.org/10.7202/027684ar

Tous droits réservés @ Département des relations industrielles de l'Université Laval, 1966
Ce document est protégé par la loi sur le droit d'auteur. L’utilisation des services d'Érudit (y compris la reproduction) est assujettie à sa politique d'utilisation que vous pouvez consulter en ligne.

https://apropos.erudit.org/fr/usagers/politique-dutilisation/ 
travailleur soit persuadé qu'en travaillant dans une industrie, il travaille au sein d'une collectivité et que, de son côté, la population l'accueillant soit convaincue que le migrant contribue d'une façon importante ou progrès et au bien-être de l'ensemble de la communauté.

Enfin, l'auteur formule des recommandations spécifiques concernant les méthodes d'adaptation et il nous invite, en les liasnt, à avoir présent à l'esprit le caractère de réciprocité du phénomène. Cette étude intéresse houtement tous ceux préoccupés par une utilisation rationnelle du potentiel humain de leur société.

\section{Jean-Paul Rheault}

White Collar Unions in Australia, R.M Martin, Monograph No 5. The Australian Institute of Political Science, Sydney, 1965, 40 pages

Cette récente publication fournira à qui aura l'heureuse idée de la consulter une information concise et assez complète sur la situation du syndicalisme des collets blancs en Australie.

Sans faire de distinctions entre les employés de bureau, les employés de services, les fonctionnaires, les cadres ou les professionnels, l'auteur se contente de décrire l'état actuel du syndicalisme pour le personnel occupant des fonctions non-manuelles.

C'est d'abord l'évolution rapide de ce genre de syndicalisme qui attire l'attention. Depuis 1880, I'année qui en marque les débuts, nous pouvons constater qu'il ne connaît que du succès dans presque tous les secteurs d'activités.

Puis après une énumération chronologique complète des différents genres d'associations qui se sont formées jusqu'à nos jours, l'auteur considère quelques données statistiques pour analyser l'évolution des ropports entre par exemple la main-d'oeuvre syndiquée en général et celle des travailleurs non manuels, et d'autres encore.

Nous passons ensuite à une étude de la répartition du « membership » des 347 syndicats actuels de l'Australie, dont 192 pour les collets blancs, sons oublier celle des divers types d'organisation que nous $y$ rencontrons (structures verticales et horizontoles) avec leurs principales caractéristiques.

Une troisième section est consacrée à l'analyse du changement qui se produit dans le caractère du syndicalisme des collets blancs.

11 est intéressant d'y remarquer la comparaison des caractères que l'auteur fait à cette occasion entre le syndicalisme des travailleurs non-manuels (attitudes du membership, identification de classe, comportement des syndicats).

Enfin c'est un bref exposé sur le militantisme chez ces syndicats lequel est d'une nature particulière chez les professionnels qui vient terminer les propos de R.M. Martin.

\section{Ronald Pleau}

\section{Le secret en matière d'inventions, André} Bertin, Editions du Tambourinaire. Entreprise Moderne d'Edition, Paris, 1965. 157 pages.

«Elément essentiel de force, de prestige, parfois même de survie des entreprises, "la recherche' entraîne des dépenses si considérables et oblige les chercheurs à de si grands efforts; que rien ne doit être négligé pour en sauvegarder les résultats, lorsqu'ils interviennent »... (préface)

L'auteur, dans ce volume, tente de cerner les différentes facettes du problème.

De sorte que I'on retrouve ou sommaire.

I - La nature des secrets en cause

2-Brevet ou secret: vaut-il mieux prenprendre un brevet ou garder le secret.

3-La protection du secret technique (know-how)

4- Le secret de fabrique

5-Questions connexes: limites légales au secret, le secret au cours de la génération de l'invention, les emprises générales exercées sur les secrets techniques

6-Comment intéresser les ingénieurs aux questions de propriété industrielle.

L'analyse qui est aussi faite du système octuel de brevets et de protection pour l'invention permet de constater l'inefficacité de la loi actuelle en France. L'auteur suggère alors un plan qui servirait à l'Europe pour les brevets et les secrets techniques. Certes le problème du secret technique est complexe mais, selon l'auteur, il est nécessaire d'avoir un véritable système de protection pour ne pas compromettre «profondément la génération et l'exploitation des inventions " (p. 147). 
Jusqu'ici le législateur donne une protection au brevet d'invention, mais non au secret technique. Et si un tel système a pu être établi pour le premier, on peut aussi le faire pour le second, en établissant un document de référence (attestant la possession personnelle) en termes de brevet.

\section{Fernand Blais}

The Rule of Law in European Integration, Stuart A. Scheingold, McGill University Press, Montréal, 1965. 331 pages.

Parmi les institutions dont nous entendons le plus parler dans l'économie européenne moderne, nous remarquons la Communauté du charbon et de l'acier (le plan Schuman), le Marché commun et l'Euratom. Or, pour que les états membres de ces institutions qui se sentent lésés dans leurs droits et qui ont des griefs à formuler à l'endroit des systèmes puissent avoir certains recours, il a fallu instituer également une cour de justice pour l'audition de ces cos.

Ainsi dans cet ouvrage l'auteur examine le rôle de cette cour et établit la croissance de son approche fonctionnelle vis-à-vis les cas économico-juridiques qui lui sont présentés. II s'en tient ici à ceux formulés dans le cadre du plan Schuman lesquels sont d'ailleurs semblables aux autres et à ceux de l'intégration régionale en général. Ces cas constituent en quelque sorte une histoire judiciaire de cette institution et fournissent un excellent terrain d'étude pour une analyse.

Scheingold essaie de voir jusqu'à quel point après dix ans d'application du système de la communauté cette cour de justice s'est ajustée avec succès à son environnement et si cette dernière a apporté une contribution véritable au succès des Communautés mentionnées.

Selon les termes de l'auteur lui-même le but de cet exposé est de renseigner sur la relation réciproque qui existe entre les aspects légal et politique de l'intégration européenne.

Les deux premiers chapitres de cette étude soulignent la tension qui existe entre les rôles politiques et judiciaires de la cour de justice. Les chapitres suivants serviront à justifier l'argument qui veut que la cour oit adopté des techniques fonctionnelles et à démontrer le succès des méthodes fonctionnelles pour imposer des règles normatives au processus d'intégration.
Mais pour comprendre complètement la contribution du fonctionalisme, l'auteur voit la nécessité d'un côté, d'explorer ses limitations et, d'un autre côté, d'établir la faiblesse des méthodes olternatives adoptées par cette cour. C'est à cette tâche qu'il se consacre dans les deux chapitres suivants de la partie II.

Lo partie III traitera de l'évolution de la technique fonctionnelle et les IVe et $\mathrm{Ve}$ parties, de ses limitations, et plus spécialement des problèmes d'intégration partielle et de changement politique. II termine enfin sur une série de conclusions des plus intéressantes.

Le tout constitue donc une analyse des problèmes de conflits entre la recherche de la stabilité et le processus dynamique de I'intégration et de l'écart entre les institutions nationales juridiques et politiques.

\section{Ronald Pleau}

The Family, William J. Goode, Foundations of Modern Sociology Series, Prentice-Hall, Inc., Englewood Cliffs, New Jersey. 1965. 120 pages

II sera de tout temps nécessaire de traiter des relations familiales et d'insister sur les résultats et découvertes de la théorie sociologique à ce sujet.

Ainsi par exemple en étudiant les relations entre les systèmes de famille et la structure sociale plus vaste, nous pouvons dire que nous ne devons plus considérer la famille comme un simple ensemble de variables dépendantes expliquées par les \& forces économiques » seules. II faut chercher les relations entre les variables centrales et s'efforcer d'explorer la structure sociale plus vaste.

Quant au problème des valeurs, nous pouvons dire que l'analyse que contient ce volume n'en fait pas une évaluation mais que la sociologie comme science peut examiner de temps en temps certains modes alternatifs de vivre.

L'cuteur divise son oeurre en dix courts chapitres et considère la famille et les systèmes de relations familiales sous des aspects très variés bien que très loggiquement relics entre eux.

Toutes les sociétés ne conçoivent pas la famille de la même façon: certaines ont même des préjugés ou sujet de cette dernière. Mais quoiqu'il en soit, la famille restera toujours une institution unique qui 